\title{
Reply to the comment on 'Nab-paclitaxel plus gemcitabine for metastatic pancreatic adenocarcinoma after Folfirinox failure: an AGEO prospective multicentre cohort'
}

\author{
Simon Pernot ${ }^{1}$, Jean-Baptiste Bachet ${ }^{2}$, Alix Portal ${ }^{1}$ and Julien Taieb* ${ }^{*}$
}

${ }^{1}$ Department of Gastroenterology and Digestive Oncology, Georges Pompidou European Hospital, Assistance Publique-Hôpitaux de Paris (AP-HP), Paris Descartes University, Paris 75015, France and ²Department of Gastroenterology, La Pitié-Salpétrière Hospital, Assistance PubliqueHôpitaux de Paris (AP-HP), Sorbonne University, UPMC University, Paris 75013, France

Sir,

We thank Vivaldi et al (2015) for their interest in reading our paper. As they noted, encouraging progression-free survival (PFS) and overall survival (OS) were found with 5.1 months and 8.8 months, respectively (Portal et al, 2015).

We argue that patients in our study were highly selected because they were able to receive a second-line therapy (CT2), even if we had more patients with an Eastern Cooperative Oncology Group (ECOG) performance status of 2 than in the MPACT trial (Von Hoff et al, 2013). Moreover, our population was selected with other characteristics: mean age was 59 years old (63 in MPACT), 63\% of patients had 1 metastatic site and only $9 \%$ had $\geqslant 3$ metastatic sites as compared with $8 \%$ and $46 \%$, respectively, in MPACT, median Ca 19.9 was $636 \mathrm{U} \mathrm{ml}^{-1}$ in our patients and $2293 \mathrm{U} \mathrm{ml}^{-1}$ in MPACT. Finally, $14 \%$ of patients had metachronous metastases after surgery (vs 7\% in MPACT) and $24.5 \%$ had previously locally advanced disease when they received FOLFIRINOX as a first-line treatment. These patients may have a better prognosis than patients with synchronous metastases.

Among patients who stopped CT2 during the follow-up period, $62.5 \%$ received a third-line chemotherapy, which reflects the selection of our population.

We agree with the remarks of Vivaldi et al (2015), concerning the necessity to identify some parameters to decide upon which subgroup may benefit of CT2. Because of the limited number of patients and already selected patients, the only significant parameter was the age of patients with a risk factor of 0.93 (95\% CI $0.87-0.99$ ).

Vivaldi et al (2015) report their prospective evaluation of mPC patients who progressed after modified FOLFIRINOX. Survival was lower than in our study despite similar characteristic, with a median PFS and OS of 2.5 months and 6.2 months, respectively. Only $18 \%$ of patients received gemcitabine + Nab-Paclitaxel, with survival in the same range. However, it suggests that all patients were not screened to receive gemcitabine + Nab-paclitaxel after progression under FOLFIRINOX, as most of patients received 5FU-based therapy, including re-challenge with FOLFIRINOX or FOLFIRI. It could lead to selection bias in the population receiving gemcitabine + Nab-paclitaxel. Moreover, the number of patients treated with gemcitabine $+\mathrm{Nab}$-paclitaxel in their study remains low and may not be used to drive any conclusion.

Vivaldi et al (2015) noted that patients were not chemorefractory, with a median PFS for first-line treatment of 5.7 months. One can hypothesise that fast progression with FOLFIRINOX might signify a chemorefractory disease and that these patients might be bad candidates for CT2. However, in our study PFS under Nab-paclitaxel + gemcitabine (PFS-2) was on the contrary significantly better, whereas PFS under firstline FOLFIRINOX (PFS-1) was lower than the median.
We recently updated our data to specifically study the link between efficiency of FOLFIRINOX in first-line treatment then efficiency of Nabpaclitaxel + gemcitabine in second-line treatment.

Sixty-one patients were included. PFS-1 was 248 days, with the rapidly progressive (RP) group having a PFS- $1<248$ days $(n=30)$ and the low progressive (LP) group a PFS-1 $\geqslant 248$ days $(n=31)$. PFS-2 was significantly increased among the RP group (median of 188 days $v s$ 144 days, HR 0.48 ; $95 \%$ CI $0.24-0.8 ; P=0.01$; Figure $1 \mathrm{~A}$ ). OS from the start of CT2 (OS-2) was also significantly increased among RP group (median 270 days vs 240 days; HR 0.5; 95\% CI 0.22-0.95; $P=0.049$; Figure 1B). Nab-paclitaxel + gemcitabine appear to be more effective in RP patients than in LP.

We have some hypotheses to explain this:

(i) Long exposure to FOLFIRINOX could lead to selection of more aggressive clones.

(ii) Long exposure to oxaliplatin can cause increased neuropathy, which may result in lower dose-intensity of Nab-paclitaxel.

(iii) The RP patients under FOLFIRINOX could be refractory to fluorouracilbased chemotherapy but not gemcitabine + Nab-paclitaxel.

Among RP patients, PFS-2 was significantly longer than PFS-1 (median 188 days $v s 98$ days, $P=0.0005$ ), supporting this last hypothesis. We can evaluate the benefit of CT2 using the growth modulation index (GMI). Using each patient as his own control, the GMI was defined as the ratio of PFS-2 to PFS-1 (Von Hoff, 1998). A GMI $>1$ indicates that PFS was longer with CT2, whereas a GMI $>1.33$ suggests that CT2 may be considered to have significant benefit. The GMI has been used in previous studies in $\mathrm{mPC}$ or colorectal cancer (Bachet et al, 2009). In our study, $24.5 \%$ and $34.4 \%$ of patients had a GMI of $>1.33$ and $>1$, respectively, reaching $50 \%$ and $70 \%$ in the RP patients, whereas none of the LP patients had a GMI of $>1$ (Figure 2).

Despite this increase in PFS-2, RP patients had still a worse OS from the start of FOLFIRINOX (OS $1+2$ ) with a median of 502 days $v s 565$ days, $(P=0.0042)$.

It would be therefore of great interest to identify patients which could have a better sensitivity to Nab-paclitaxel + gemcitabine than to FOLFIRINOX. Molecular marker should be validated as expression of HENT-1 as a marker of efficacy of gemcitabine-based chemotherapy (Maréchal et al, 2012).

However, to date, no biomarker has been validated, and a sequential regimen alternating 5FU-based and gemcitabine-based therapies might allow to use precociously two potentially efficient regimens. The sequential strategy was first tested in $\mathrm{MPC}$ with alternating 2
A

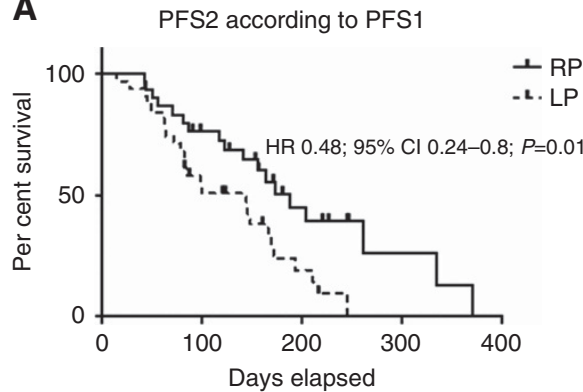

B

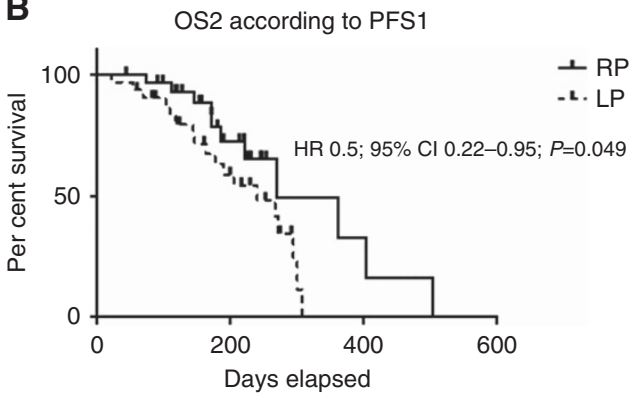

Figure 1. Survival under second-line chemotherapy with gemcitabine + Nab-paclitaxel, according to first-line PFS. (A) PFS under second-line chemotherapy with gemcitabine + Nab-paclitaxel (PFS-2): RP patients vs low progressive patients. (B) OS under second-line chemotherapy with gemcitabine + Nab-paclitaxel (OS-2): RP patients vs low progressive patients. 
A

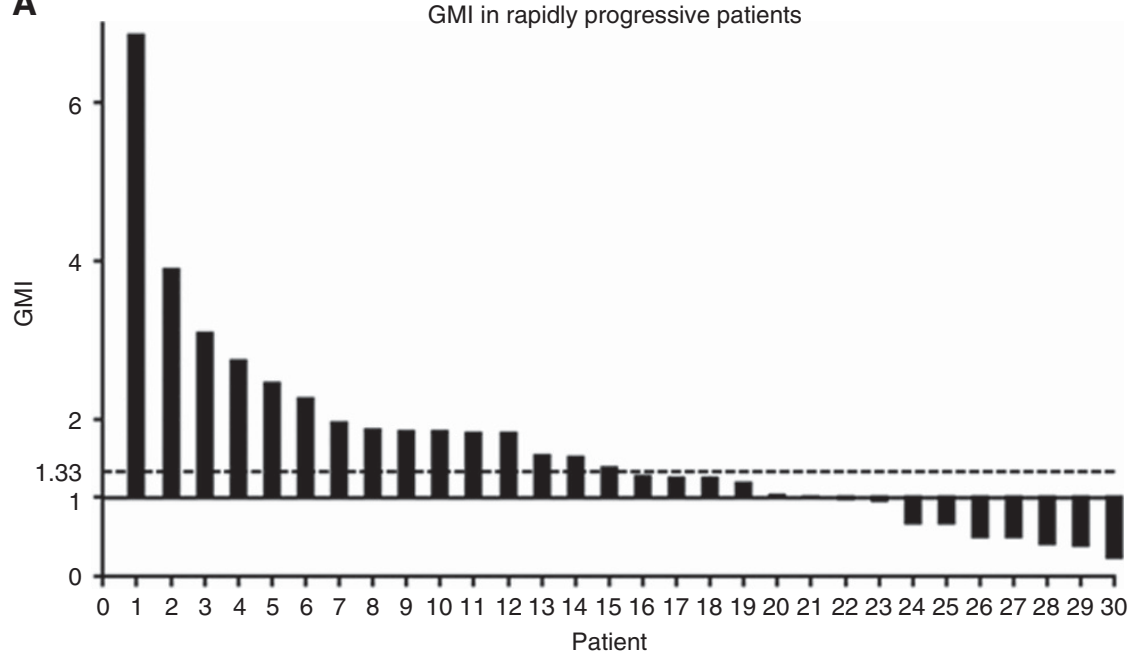

B

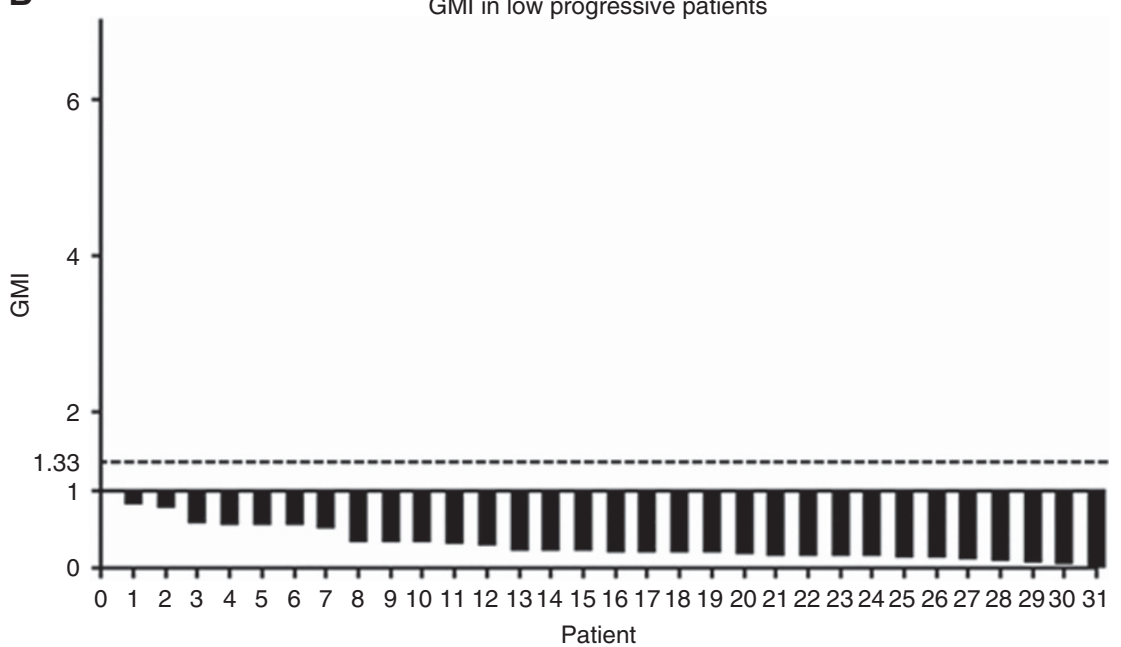

Figure 2. Growth modulation index. (A) Rapidly progressive (RP) patients and (B) low progressive (LP) patients.

months of gemcitabine and 2 months of FOLFIRI-3 (Trouilloud et al, 2014), which resulted in increased PFS and OS compared with gemcitabine. This regimen is currently being tested $v$ s FOLFIRINOX (PRODIGE 35, NCT02352337), and has been extrapolated with gemcitabine + Nab-paclitaxel in the FIRGEMAX and the GABRINOX trial (NCT01964287). In these ongoing trials, patients receive gemcitabine + Nab-paclitaxel alternating with FOLFIRI-3 or FOLFIRINOX, respectively.

In conclusion, we think that clinical parameters to choose the right patient for gemcitabine + Nab-paclitaxel after failure of FOLFIRINOX could include a limited number of metastatic site, metachronous metastases, and low Ca 19.9 level. Fast progression under FOLFIRINOX in first-line treatment is predictive of efficacy of gemcitabine $+\mathrm{Nab}$ paclitaxel. However, these observations should be confirmed with prospective data and research for validated biomarkers remains an emergency to optimise mPC treatment.

\section{CONFLICT OF INTEREST}

The authors declare no conflict of interest.

\section{REFERENCES}

Bachet J-B, Mitry E, Lièvre A, Lepère C, Vaillant J-N, Declety G, Parlier H, Emile J-F, Julié C, Rougier P (2009) Second- and third-line chemotherapy in patients with metastatic pancreatic adenocarcinoma: feasibility and potential benefits in a retrospective series of 117 patients. Gastroenterol Clin Biol 33: 1036-1044.

Maréchal R, Bachet J-B, Mackey JR, Dalban C, Demetter P, Graham K, Couvelard A, Svrcek M, Bardier-Dupas A, Hammel P, Sauvanet A, Louvet C, Paye F, Rougier P, Penna C, André T, Dumontet C, Cass CE, Jordheim LP, Matera E-L, Closset J, Salmon I, Devière J, Emile J-F, Van Laethem J-L (2012) Levels of gemcitabine transport and metabolism proteins predict survival times of patients treated with gemcitabine for pancreatic adenocarcinoma. Gastroenterology 143: 664-674.

Portal A, Pernot S, Tougeron D, Arbaud C, Bidault AT, de la Fouchardière C, Hammel P, Lecomte T, Dréanic J, Coriat R, Bachet JB, Dubreuil O, Marthey L, Dahan L, Tchoundjeu B, Locher C, Lepère C, Bonnetain F, Taieb J (2015) Nab-paclitaxel plus gemcitabine for metastatic pancreatic adenocarcinoma after Folfirinox failure: an AGEO prospective multicentre cohort. Br J Cancer 113(7): 989-995.

Trouilloud I, Dupont-Gossard A-C, Malka D, Artru P, Gauthier M, Lecomte T, Aparicio T, Thirot-Bidault A, Lobry C, Asnacios A, Manet-Lacombe S, Fein F, Dubreuil O, Landi B, Zaanan A, Bonnetain F, Taïeb J (2014) Fixed-dose rate gemcitabine alone or alternating with FOLFIRI.3 (irinotecan, leucovorin and fluorouracil) in the first-line treatment of patients with metastatic pancreatic adenocarcinoma: an AGEO randomised phase II study (FIRGEM). Eur J Cancer 50: 3116-3124.

Vivaldi C, Caparello C, Pasquini G, Musettini G, Catanese S, Lencioni M, Falcone A, Vasile E (2015) Second-line treatment after disease progression following first-line chemotherapy with modified FOLFIRINOX in advanced pancreatic cancer patients: a single institution retrospective cohort study. Ann Oncol Off J Eur Soc Med Oncol 26(Supp 4): iv52.

Von Hoff DD (1998) There are no bad anticancer agents, only bad clinical trial designs-twenty-first Richard and Hinda Rosenthal Foundation Award Lecture. Clin Cancer Res Off J Am Assoc Cancer Res 4: $1079-1086$. 
Von Hoff DD, Ervin T, Arena FP, Chiorean EG, Infante J, Moore M, Seay T, Tjulandin SA, Ma WW, Saleh MN, Harris M, Reni M, Dowden S, Laheru D, Bahary N, Ramanathan RK, Tabernero J, Hidalgo M, Goldstein D, Van
Cutsem E, Wei X, Iglesias J, Renschler MF (2013) Increased survival

in pancreatic cancer with nab-paclitaxel plus gemcitabine. $N$ Engl J Med 369: 1691-1703.

*Correspondence: Professor J Taieb; E-mail: jtaieb75@gmail.com

Published online 28 April 2016

(c) 2016 Cancer Research UK. All rights reserved 0007-0920/16 